\title{
Expanded Chemistry of Formamidine Ureas
}

\author{
David D. Díaz and M.G. Finn*
}

Department of Chemistry and The Skaggs Institute for Chemical Biology, The Scripps Research Institute, 10550 N. Torrey Pines Rd., La Jolla, CA 92037 USA

\section{Supporting Information}

General. ${ }^{1} \mathrm{H}$ and ${ }^{13} \mathrm{C}$ NMR spectra were recorded on Bruker DRX-500, AMX-400, or Varian Mercury 200 spectrometers, using $\mathrm{CDCl}_{3}$ as solvent. Mass spectra were obtained on an Agilent $1100 \mathrm{LC} / \mathrm{MS}$ spectrometer (model G1946A) with mobile phase composed of 90:10 $\mathrm{CH}_{3} \mathrm{OH}: \mathrm{H}_{2} \mathrm{O}$ containing $0.1 \% \mathrm{CF}_{3} \mathrm{CO}_{2} \mathrm{H}$. Melting points were measured in a Thomas Hoover capillary melting point apparatus and are uncorrected. Infrared spectra were recorded on a MIDAC EM200 instrument with horizontal attenuated total reflectance accessory from Pike Instruments. Column chromatography was performed on EM Science silica gel, $60 \AA$ and $40 \square 63 \square \mathrm{m}$. TLC analysis was facilitated by the use of the following stains in addition to UV light with fluorescent-indicating plates: phosphomolybdic acid, vanillin/EtOH, anisaldehyde/EtOH, or $\mathrm{KMnO}_{4} / \mathrm{H}_{2} \mathrm{O} . \mathrm{CH}_{2} \mathrm{Cl}_{2}$ was dried by passage through activated alumina column; ${ }^{1}$ dry $\mathrm{Et}_{3} \mathrm{~N}$ and the others solvents used in this work were purchased from Aldrich. Reactions requiring anhydrous conditions were performed under nitrogen. Anhydrous magnesium sulfate was used for drying solutions.

General procedure for thiolcarbamate formation.

To a solution of 1-(tert-butylimino-methyl)-1,3-dimethyl-urea hydrochloride (1b) $(2.4$ mmol, 1.0 equiv) in dry THF $(25 \mathrm{~mL})$ at room temperature under a nitrogen atmosphere was added $\mathrm{Et}_{3} \mathrm{~N}$ ( $2.7 \mathrm{mmol}, 1.15$ equiv). The reaction mixture was stirred for $5 \mathrm{~min}$, after which the corresponding thiol (3.6 mmol, 1.5 equiv) was added. The reaction mixture was refluxed for 12 $\mathrm{h}$, cooled, filtered to remove solid material, and evaporated under vacuum. The residue was purified by column chromatography to yield the thiolcarbamate derivative.

Solvents other than THF and acetonitrile may be used, such as toluene, $\mathrm{Et}_{2} \mathrm{O}$, acetone, or DMF, although lower yields were obtained, and the reaction does proceed well neat (without solvent). At room temperature, it is very sluggish in any solvent. It should be noted that the reactions of $\mathbf{1 b}$ with 4-nitrobenzenethiol and pentachlorothiophenol gave inseparable mixtures of products.

General procedure for $N$-alkylation/N-acylation of formamidine ureas and reactions with isocyanates.

To a solution of the formamidine urea $(1.0 \mathrm{mmol})$ in dry THF $(0.1 \mathrm{M})$ cooled at $-78^{\circ} \mathrm{C}$ under a nitrogen atmosphere was added $n$-BuLi (1.6M solution in hexanes, $0.98 \mathrm{mmol})$ dropwise. The reaction mixture was stirred for $5 \mathrm{~min}$ at $-78{ }^{\circ} \mathrm{C}$ and then $15 \mathrm{~min}$ at $0{ }^{\circ} \mathrm{C}$. After this time the mixture was cooled again at $-78{ }^{\circ} \mathrm{C}$ and the electrophile $(2.0 \mathrm{mmol})$ was added dropwise. The reaction mixture was allowed to warm to room temperature over ca. $1.5 \mathrm{~h}$ and stirred for a further $20 \mathrm{~h}$. The reaction mixture was quickly partitioned between $\mathrm{Et}_{2} \mathrm{O}$ and

1) Pangborn, A. B.; Giardello, M. A.; Grubbs, R. H.; Rosen, R. K.; Timmers, F. J. Organometallics 1996, 15, $1518 \square 1520$. 
saturated aqueous $\mathrm{NH}_{4} \mathrm{Cl}$, washed quickly with brine, dried $\left(\mathrm{MgSO}_{4}\right)$, filtered, concentrated, and purified by column chromatography to yield the corresponding formamidine derivative.

With isocyanates $\mathrm{R}^{2} \mathrm{NCO}$, the derived urea $\left(\mathrm{R}^{2} \mathrm{NHCONHR}{ }^{2}\right)$ was always found as a significant byproduct, in addition to many other species observed on TLC. No reaction was observed in the absence of base, even at reflux. The expected acylated structure $\mathbf{1 3}$ was never observed. The yields of products $\mathbf{1 0}$ were increased by approximately $10 \%$ when three equivalents of isocyanate was used. Because of the complex nature of the reaction mixtures, we cannot rule out the formation of both isomers of 10 (10 and iso-10, Scheme 5). Indeed, the structure of the major isomer is given based on the similarity of the N-Me chemical shift to other well-characterized formamidine ureas, but should not be regarded as firmly established.

Crossover Experiment (Figure 1). A solution of compound $\mathbf{1 b}(6.2 \mathrm{mg}, 0.043 \mathrm{mmol})$ and $\mathbf{1 d}$ $(10.0 \mathrm{mg}, 0.043 \mathrm{mmol})$ in $1 \mathrm{~mL}$ THF were treated at room temperature with $0.064 \mathrm{mmol}(0.74$ equiv.) $n$-BuLi. After 30 minutes, the reaction mixture was analyzed by TLC. The two most intense spots corresponded to the starting materials; two other clear spots showed identical $\mathrm{R}_{\mathrm{f}}$ 's to $1 \mathbf{a}$ and 1e, which was verified by co-spotting with authentic materials. Mass spectrometry analysis (direct injection to an Agilent $1100 \mathrm{MSD}$ instrument, with acetonitrile/0.2\% TFA mobile phase) is shown in Figure S1.

Infrared Spectroscopy Analysis of Formamidine-Urea + Base (Figure 1). To further establish the proposed base-mediated fragmentation of formamidine ureas, the reaction of compound with $n$-BuLi was followed by IR, as shown in Figure S2. A well-defined band was observed in the characteristic isocyanate region $\left(2227 \mathrm{~cm}^{-1}\right)$.

Compound characterization. Commercially available $\mathbf{2 a}$ and $\mathbf{2 e}$ were found to be identical to the material produced by the reaction of $\mathbf{1 b}$ with the appropriate thiophenol. Compounds $\mathbf{2} \mathbf{b},{ }^{2} \mathbf{2 d},{ }^{3}$ $\mathbf{2} \mathbf{i},{ }^{4}$ and $\mathbf{4}^{\mathbf{5}}$ have been previously described.

Methylthiocarbamic acid $S$-(4-methoxyphenyl) ester (2c). White solid: Mp 90 $\square 91{ }^{\circ} \mathrm{C}$; ${ }^{1} \mathrm{H}$ NMR $\left(\mathrm{CDCl}_{3}\right) \square 2.86(\mathrm{~s}, 3 \mathrm{H}), 3.87$ (s, 3H), 5.57 (br s, 1H), 6.99 (d, $\left.J=7.3 \mathrm{~Hz}, 2 \mathrm{H}\right), 7.53$ (d, $J=7.6$ $\mathrm{Hz}, 2 \mathrm{H}) ;{ }^{13} \mathrm{C} \mathrm{NMR}\left(\mathrm{CDCl}_{3}\right) \square 28.4,55.8,115.6,119.6,137.7,161.4,168.2$; IR (thin film, $\mathrm{cm}^{-1}$ ) 2838, 2053, 1888, 1305, 999, 818; MS $m / z$ (relative intensity) $199(\mathrm{M}+2)^{+}(11), 198(\mathrm{M}+1)^{+}$ (100). HRMS calcd for $\mathrm{C}_{9} \mathrm{H}_{12} \mathrm{NO}_{2} \mathrm{~S} 198.0583$, found 198.0582 .

$S$-(4-hydroxyphenyl) methylthiolcarbamate (2f). White semisolid: ${ }^{1} \mathrm{H}$ NMR $\left(\mathrm{CDCl}_{3}\right) \square 2.90$ (d, $J$ $=4.3 \mathrm{~Hz}, 3 \mathrm{H}), 5.49($ br s, $1 \mathrm{H}), 6.76(\mathrm{~d}, J=5.1 \mathrm{~Hz}, 2 \mathrm{H}), 7.34(\mathrm{~d}, J=5.1 \mathrm{~Hz}, 2 \mathrm{H}), 8.45(\mathrm{br} \mathrm{s}, 1 \mathrm{H})$; ${ }^{13} \mathrm{C} \mathrm{NMR}\left(\mathrm{CDCl}_{3}\right) \square 28.6,117.4,118.0,137.9,158.6,168.1$; IR (thin film, $\left.\mathrm{cm}^{-1}\right) 3024,2870$, $1660,1591,1487,1221,1004,827$; MS m/z (relative intensity) $206(\square+\mathrm{Na})^{+}(32), 185(\mathrm{M}+2)^{+}$ (10), $184(\mathrm{M}+1)^{+}(100)$. HRMS calcd for $\mathrm{C}_{8} \mathrm{H}_{10} \mathrm{NO}_{2} \mathrm{~S} 184.0427$, found 184.0427.

2) Uldrich, H.; Tucker, B.; Sayigh, A. A. R. J. Org. Chem. 1967, 32, $3938 \square 3941$.

3) Rinehart, J. K. (PPG Industries, Inc.), 1976, US Patent \# 4055657.

4) Wachter, M. P. (Ortho Pharmaceutical Corp., USA), 1978, US Patent \# 4066681.

5) (a) Almqvist, F.; Guillaume, D.; Hultgren, S. J.; Marshall, G. R. Tetrahedron Lett. 1998, 39, $2293 \square 2294$.

(b) Schmitz, W. D.; Romo, D. Tetrahedron Lett. 1996, 37, 4857-4860. 
Figure S1. Mass spectrometry of formamidine urea crossover. The inset shows an independent spectrum, focusing on a narrow mass range encompassing $1 \mathbf{a}$ and $\mathbf{1 e .}$<smiles>CON=CN(C)C(=O)NC(=O)N(C)OC</smiles>

$1 b$

1d

$1 a$

$1 e$

$\mathrm{C}_{5} \mathrm{H}_{11} \mathrm{~N}_{3} \mathrm{O}_{2}$

$\mathrm{C}_{13} \mathrm{H}_{19} \mathrm{~N}_{3} \mathrm{O}$

$\mathrm{C}_{8} \mathrm{H}_{17} \mathrm{~N}_{3} \mathrm{O}$

$\mathrm{C}_{10} \mathrm{H}_{13} \mathrm{~N}_{3} \mathrm{O}_{2}$

Exact Mass: 145.09 Exact Mass: 233.15

Exact Mass: 171.14 Exact Mass: 207.10

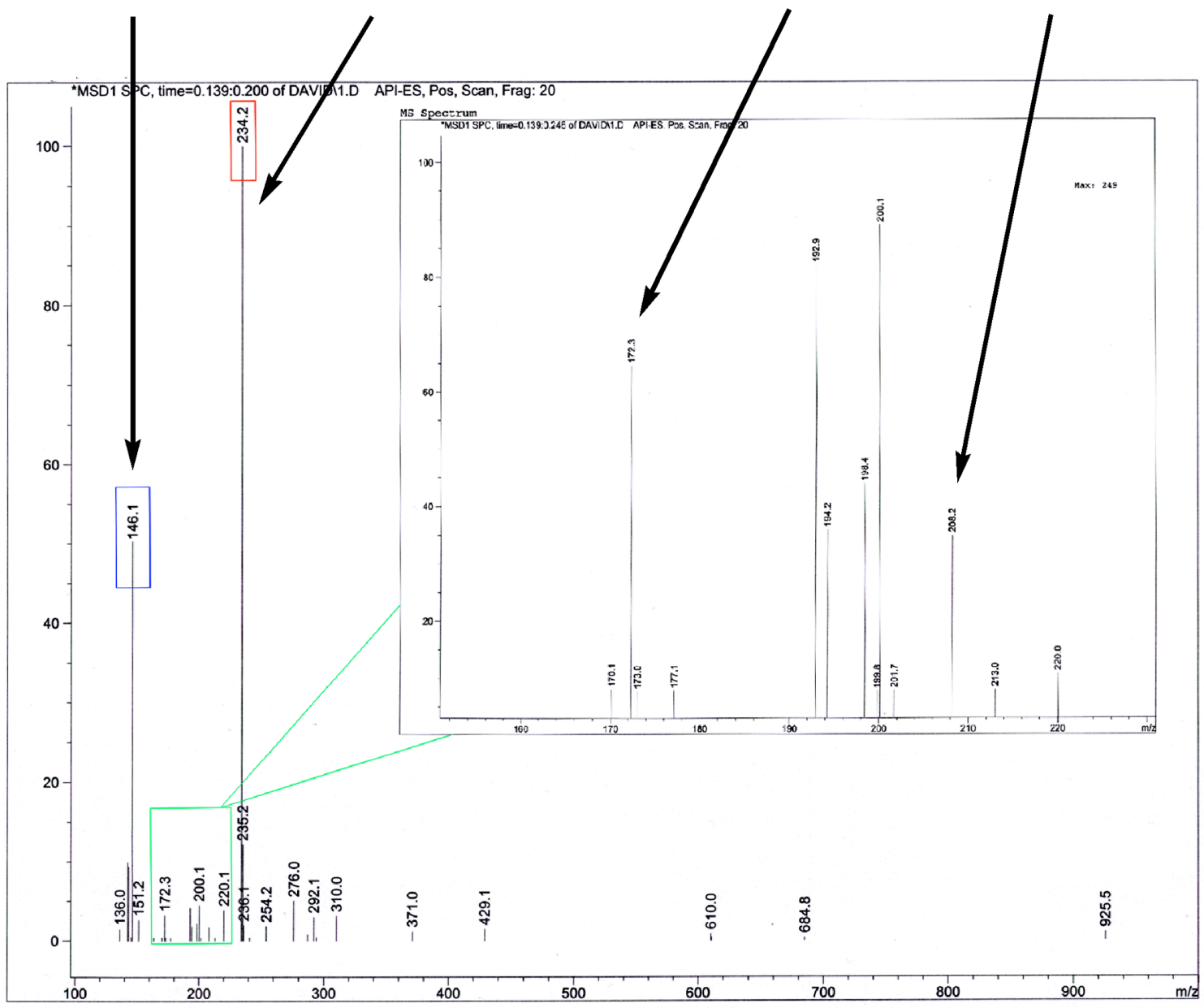


Figure S2. IR spectroscopic analysis of the treatment of compound 1a with $n$-BuLi, suggesting the production of methyl isocyanate. The bottom spectrum is that of an analogous alkyl isocyanate, showing the strong NCO stretching band. THF $+n$-BuLi alone show no bands in this region.

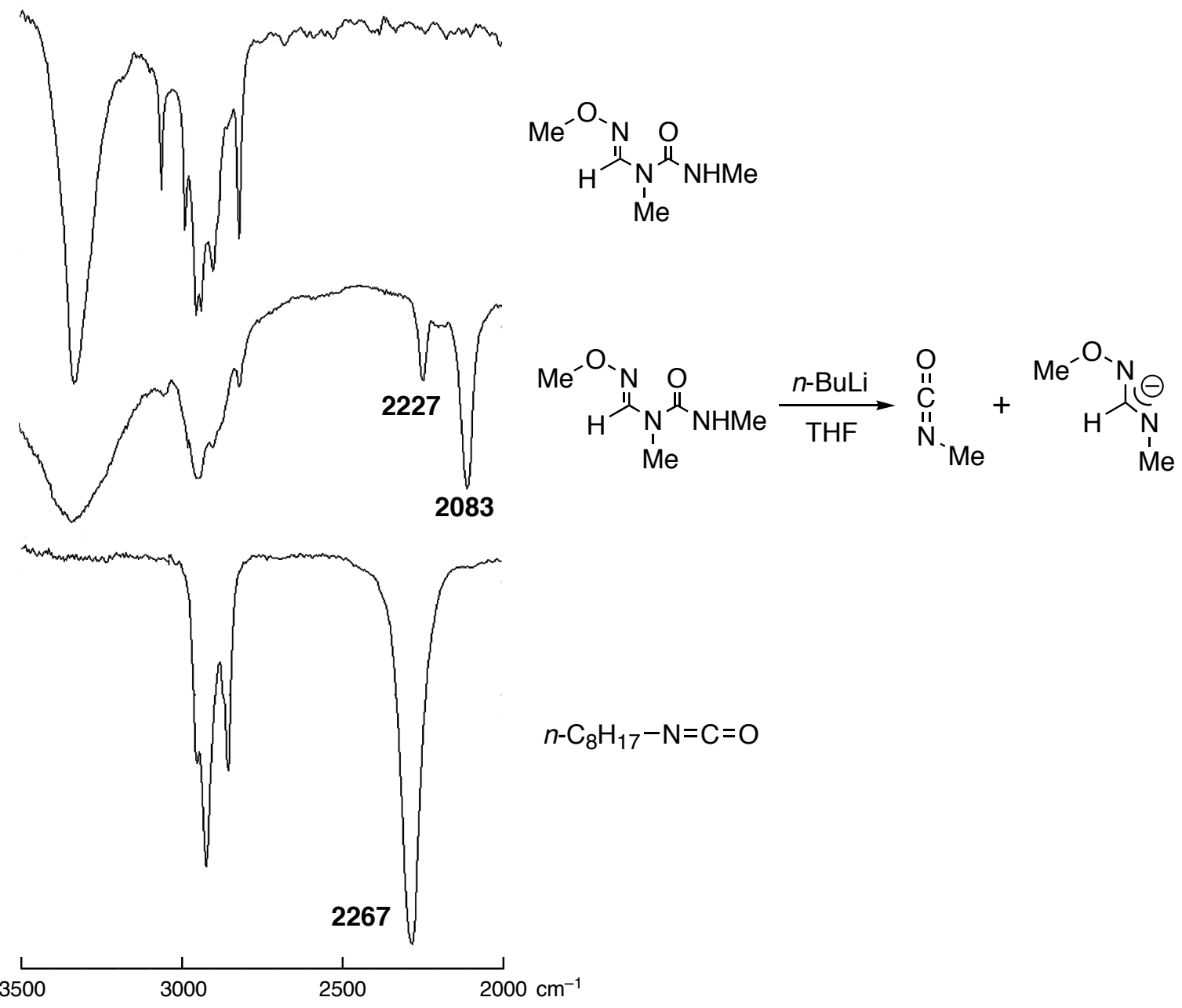

$S$-(2-naphthyl) methylthiolcarbamate (2g). White solid: Mp 94 $\square 95{ }^{\circ} \mathrm{C} ;{ }^{1} \mathrm{H}$ NMR $\left(\mathrm{CDCl}_{3}\right) \square 2.89$

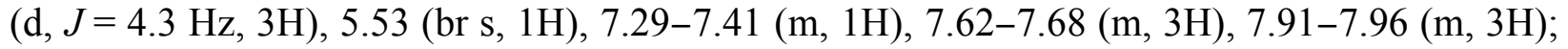
${ }^{13} \mathrm{C} \mathrm{NMR}\left(\mathrm{CDCl}_{3}\right) \square 28.5,126.2,127.3,127.9,128.2,128.4,129.7,132.2,133.8,134.0,136.0$, 167.3; IR (thin film, $\mathrm{cm}^{-1}$ ) 3278, 3053, 2940, 1680, 1499, 1338, 1226, 1008, 815; MS m/z (relative intensity) $240(\square+\mathrm{Na})^{+}(11), 219(\mathrm{M}+2)^{+}(15), 218(\mathrm{M}+1)^{+}(100)$. HRMS calcd for $\mathrm{C}_{12} \mathrm{H}_{12} \mathrm{NOS} 218.0634$, found 218.0631 .

$S$-(2-phenylethyl) methylthiolcarbamate (2h). Light yellow oil: ${ }^{1} \mathrm{H}$ NMR $\left(\mathrm{CDCl}_{3}\right) \square 2.89$ (d, $J=$ $4.4 \mathrm{~Hz}, 3 \mathrm{H}), 2.94(\mathrm{t}, J=8.4 \mathrm{~Hz}, 2 \mathrm{H}), 3.18$ (t, $J=7.4 \mathrm{~Hz}, 2 \mathrm{H}), 5.29$ (br s, 1H), $7.23 \square 7.31$ (m, $5 \mathrm{H}) ;{ }^{13} \mathrm{C} \mathrm{NMR}\left(\mathrm{CDCl}_{3}\right) \square 28.4,31.7,37.4,126.9,128.9,129.1,140.7,168.4$; IR (thin film, $\mathrm{cm}^{-1}$ ) 
$3212,3084,2934,1670,1630,1485,1223,1018,821$; MS $m / z$ (relative intensity) $218(\square+\mathrm{Na})^{+}$ (4), $197(\mathrm{M}+2)^{+}(11), 196(\mathrm{M}+1)^{+}(100)$. HRMS calcd for $\mathrm{C}_{10} \mathrm{H}_{14} \mathrm{NOS} 196.0791$, found 196.0794.

$S$-(3-chloropropyl) methylthiolcarbamate (2k). Colorless oil: ${ }^{1} \mathrm{H}$ NMR $\left(\mathrm{CDCl}_{3}\right) \square 2.07$ (q, $J=$ $6.6 \mathrm{~Hz}, 2 \mathrm{H}), 2.87(\mathrm{~d}, J=4.8 \mathrm{~Hz}, 3 \mathrm{H}), 3.05(\mathrm{t}, J=6.8 \mathrm{~Hz}, 2 \mathrm{H}), 3.62(\mathrm{t}, J=4.2 \mathrm{~Hz}, 2 \mathrm{H}), 5.36(\mathrm{br}$ $\mathrm{s}, 1 \mathrm{H}) ;{ }^{13} \mathrm{C} \mathrm{NMR}\left(\mathrm{CDCl}_{3}\right) \square 27.3,28.3,33.5,43.8,167.9$; IR (thin film, $\mathrm{cm}^{-1}$ ) 3308, 2946, 1698, 1634, 1497, 1231, 1010, 813; MS m/z (relative intensity) $190(\square+\mathrm{Na})^{+}(7), 169(\mathrm{M}+2)^{+}(35), 168$ $(\mathrm{M}+1)^{+}(100)$.

$S$-hexyl methylthiolcarbamate (2j). Colorless oil: ${ }^{1} \mathrm{H}$ NMR $\left(\mathrm{CDCl}_{3}\right) \square 0.88(\mathrm{t}, J=7.7 \mathrm{~Hz}, 3 \mathrm{H})$, $1.27 \square 1.43(\mathrm{~m}, 6 \mathrm{H}), 1.54 \square 1.65(\mathrm{~m}, 2 \mathrm{H}), 2.87(\mathrm{~d}, J=4.2 \mathrm{~Hz}, 3 \mathrm{H}), 2.88 \square 2.94(\mathrm{~m}, 2 \mathrm{H}), 5.30$ (br s, $1 \mathrm{H}) ;{ }^{13} \mathrm{C} \mathrm{NMR}\left(\mathrm{CDCl}_{3}\right) \square 14.4,22.9,28.8,28.3,30.4,30.9,31.7,168.5$; IR (thin film, $\mathrm{cm}^{-1}$ ) $3312,2938,2861,1662,1509,1219,1018,821$; MS $m / z$ (relative intensity) $198(\square+\mathrm{Na})^{+}(8)$, $177(\mathrm{M}+2)^{+}(11), 176(\mathrm{M}+1)^{+}(100)$.

Ethyl 3-\{[(methylamino)carbonyl] sulfanyl $\}$ propanoate (2l). Light yellow oil: ${ }^{1} \mathrm{H}$ NMR $\left(\mathrm{CDCl}_{3}\right)$ $\square 1.26(\mathrm{t}, J=11.2 \mathrm{~Hz}, 3 \mathrm{H}), 2.68(\mathrm{t}, J=11.2 \mathrm{~Hz}, 2 \mathrm{H}), 2.87(\mathrm{~d}, J=5.2 \mathrm{~Hz}, 3 \mathrm{H}), 3.15(\mathrm{t}, J=7.4$ $\mathrm{Hz}, 2 \mathrm{H}), 4.15$ (c, $J=7.2 \mathrm{~Hz}, 2 \mathrm{H}), 5.31$ (br s, $1 \mathrm{H}) ;{ }^{13} \mathrm{C} \mathrm{NMR}\left(\mathrm{CDCl}_{3}\right) \square$ 14.4, 25.2, 28.1, 35.8, 61.0, 167.7, 172.3; IR (thin film, $\mathrm{cm}^{-1}$ ) 3224, 2950, 1734, 1658, 1517, 1215, 1014, 809; MS m/z (relative intensity) $214(\square+\mathrm{Na})^{+}(66), 193(\mathrm{M}+2)^{+}(9), 192(\mathrm{M}+1)^{+}(100)$. HRMS calcd for $\mathrm{C}_{7} \mathrm{H}_{14} \mathrm{NO}_{3} \mathrm{~S}$ 192.0689, found 192.0687 .

$S$-(4-hydroxybutyl) methylthiolcarbamate (2m). Light yellow oil: ${ }^{1} \mathrm{H}$ NMR $\left(\mathrm{CDCl}_{3}\right) \square 1.63 \square 1.73$ $(\mathrm{m}, 4 \mathrm{H}), 2.87(\mathrm{~d}, J=4.7 \mathrm{~Hz}, 3 \mathrm{H}), 2.95(\mathrm{t}, J=6.3 \mathrm{~Hz}, 2 \mathrm{H}), 3.68(\mathrm{t}, J=6.3 \mathrm{~Hz}, 2 \mathrm{H}), 5.34($ br s, $1 \mathrm{H}) ;{ }^{13} \mathrm{C}$ NMR $\left(\mathrm{CDCl}_{3}\right) \square 27.2,28.1,29.8,31.8,62.0,168.9$; IR (thin film, $\mathrm{cm}^{-1}$ ) 3296, 2918, $1082,1634,1497,1312,1235,1002,809 ; \mathrm{MS} m / z$ (relative intensity) $173(\mathrm{M}+2)^{+}(11), 172$ $(\mathrm{M}+1)^{+}(100)$.

$S$-[2-(butylamino)ethyl] methylthiolcarbamate (2n). Light yellow oil: ${ }^{1} \mathrm{H}$ NMR $\left(\mathrm{CDCl}_{3}\right) \square 0.94$ $(\mathrm{t}, J=7.4 \mathrm{~Hz}, 3 \mathrm{H}), 1.30 \square 1.43(\mathrm{~m}, 2 \mathrm{H}), 1.44 \square 1.57(\mathrm{~m}, 2 \mathrm{H}), 2.63 \square 2.74(\mathrm{~m}, 2 \mathrm{H}), 2.80(\mathrm{~d}, J=4.6$ $\mathrm{Hz}, 3 \mathrm{H}), 3.18(\mathrm{t}, J=6.8 \mathrm{~Hz}, 2 \mathrm{H}), 3.41(\mathrm{t}, J=7.0 \mathrm{~Hz}, 2 \mathrm{H}), 4.36$ (br s, $1 \mathrm{H}), 4.44$ (br s, $1 \mathrm{H}) ;{ }^{13} \mathrm{C}$ NMR $\left(\mathrm{CDCl}_{3}\right) \square 14.3,20.5,27.9,29.9,31.1,48.1,51.4,158.9$; IR (thin film, $\left.\mathrm{cm}^{-1}\right) 3348,2958$, 2873, 1666, 1618, 1489, 1296, 1240, 946; MS m/z (relative intensity) $192(\mathrm{M}+2)^{+}(10), 191$ $(\mathrm{M}+1)^{+}(100)$. HRMS calcd for $\mathrm{C}_{8} \mathrm{H}_{19} \mathrm{~N}_{2} \mathrm{OS}$ 191.1213, found 191.1212.

$S$-(8-sulfanyloctyl) methylthiolcarbamate (2o). White solid: Mp $43 \square 44{ }^{\circ} \mathrm{C} ;{ }^{1} \mathrm{H}$ NMR $\left(\mathrm{CDCl}_{3}\right) \square$ $1.30 \square 1.35(\mathrm{~m}, 8 \mathrm{H}), 1.60(\mathrm{~m}, 4 \mathrm{H}), 2.52(\mathrm{~m}, 2 \mathrm{H}), 2.88(\mathrm{~d}, J=4.8 \mathrm{~Hz}, 3 \mathrm{H}), 2.89 \square 2.95(\mathrm{~m}, 2 \mathrm{H})$, 5.29 (br s, $1 \mathrm{H}) ;{ }^{13} \mathrm{C} \mathrm{NMR}\left(\mathrm{CDCl}_{3}\right) \square 25.0,28.2$, 28.6, 29.0, 29.3, 29.4, 30.3, 30.9, 34.3, 168.5; IR (thin film, $\mathrm{cm}^{-1}$ ) 3336, 2930, 2853, 1646, 1529, 1465, 1227, 821; MS $\mathrm{m} / z$ (relative intensity) $258(\square+\mathrm{Na})^{+}(8), 237(\mathrm{M}+2)^{+}(13), 236(\mathrm{M}+1)^{+}(100)$. HRMS calcd for $\mathrm{C}_{10} \mathrm{H}_{22} \mathrm{NOS}_{2} 236.1137$, found 236.1134 . 
Thiazoline 4 was reduced to the corresponding hydroxymethyl compound by $\mathrm{NaBH}_{4}$ as previously described. $^{5 \mathrm{~b}}$ The compound we obtained showed $[\square]_{\mathrm{D}}{ }^{25}=+89^{\circ}$, identical within experimental error to the reported value of $+93^{\circ} .^{5 b}$

(4R,5R)-4,5-Diphenyl-4,5-dihydro-1H-imidazole (8). To a solution of 1-(tert-butyliminomethyl)-1,3-dimethyl-urea hydrochloride $(207 \mathrm{mg}, 1.0 \mathrm{mmol})$ in dry $\mathrm{CH}_{2} \mathrm{Cl}_{2}(2 \mathrm{~mL}, 0.5 \mathrm{M}$ in formamidine) at room temperature under a nitrogen atmosphere was added $\mathrm{Et}_{3} \mathrm{~N}$ (156 _ L, 1.1 mmol). The reaction mixture was stirred for $5 \mathrm{~min}$, after which time was heated at $50{ }^{\circ} \mathrm{C}$ and $(1 R, 2 R)-(+)-1,2$-diphenylethylenediamine $(318 \mathrm{mg}, 1.5 \mathrm{mmol})$ was added. The reaction mixture was refluxed for $3 \mathrm{~h}$, after which time the solvent was evaporated. The residue was triturated with THF, filtered, concentrated and recrystallized from ethyl acetate to afford $\mathbf{8}$ in $62 \%$ yield. Colorless solid: $\mathrm{Mp} 99 \square 101^{\circ} \mathrm{C}$; $[\square]^{20}{ }_{\mathrm{D}}+341^{\circ}\left(c=0.63, \mathrm{CH}_{3} \mathrm{OH}\right){ }^{1} \mathrm{H}$ NMR $\left(\mathrm{CDCl}_{3}\right) \square 4.71(\mathrm{~s}, 2 \mathrm{H})$, $7.26(\mathrm{~d}, J=7.0 \mathrm{~Hz}, 4 \mathrm{H}), 7.30(\mathrm{~d}, J=6.6 \mathrm{~Hz}, 2 \mathrm{H}), 7.35(\mathrm{~d}, J=7.0 \mathrm{~Hz}, 4 \mathrm{H}) ;{ }^{13} \mathrm{C} \mathrm{NMR}\left(\mathrm{CDCl}_{3}\right) \square$ 126.9, 127.9, 129.1, 143.6, 153.9; IR (thin film, $\mathrm{cm}^{-1}$ ) 3346, 2920, 1631, 1581, 1410, 1276, 1179; MS $m / z$ (relative intensity) $224(\mathrm{M}+2)^{+}(18), 223(\mathrm{M}+1)^{+}(100)$. HRMS calcd for $\mathrm{C}_{15} \mathrm{H}_{15} \mathrm{~N}_{2}$ 223.1230, found 223.1230. The meso form of this compound has been described. ${ }^{6}$

1-(tert-Butylimino-methyl)-1,3,3-trimethyl urea (9a). Colorless oil: ${ }^{1} \mathrm{H}$ NMR $\left(\mathrm{CDCl}_{3}\right) \square 1.20$ (s, 9H), 2.90 (s, 3H), 3.00 (s, 3H), 3.09 (s, 3H), 7.96 (br s, $1 \mathrm{H}) ;{ }^{13} \mathrm{C}$ NMR $\left(\mathrm{CDCl}_{3}\right) \square 31.1$, 32.6, 38.7, 38.9, 54.9, 147.5, 163.4; IR (thin film, $\mathrm{cm}^{-1}$ ) 3480, 2926, 1665, 1487, 1373, 1306, 1115; MS $m / z$ (relative intensity) $187(\mathrm{M}+2)^{+}(10), 186(\mathrm{M}+1)^{+}(100)$. HRMS calcd for $\mathrm{C}_{9} \mathrm{H}_{20} \mathrm{~N}_{3} \mathrm{O}$ 186.1601, found 186.1601 .

1-(tert-Butylimino-methyl)-1,3-dimethyl-3-octyl urea (9b). Colorless oil: ${ }^{1} \mathrm{H}$ NMR $\left(\mathrm{CDCl}_{3}\right) \square$ $0.91(\mathrm{t}, J=6.9 \mathrm{~Hz}, 3 \mathrm{H}), 1.24(\mathrm{~s}, 3 \mathrm{H}), 1.24 \square 1.33(\mathrm{~m}, 12 \mathrm{H}), 2.92(\mathrm{~s}, 3 \mathrm{H}), 3.12(\mathrm{~s}, 3 \mathrm{H}), 3.27(\mathrm{t}, J=$ $6.6 \mathrm{~Hz}, 2 \mathrm{H}), 7.98$ (br s, $1 \mathrm{H}) ;{ }^{13} \mathrm{C}$ NMR $\left(\mathrm{CDCl}_{3}\right) \square 14.5,23.0,27.1,27.7,29.6,31.1,32.1,32.7$, 36.6, 50.9, 54.9, 147.6, 162.2; IR (thin film, $\mathrm{cm}^{-1}$ ) 3438, 2935, 2862, 1657, 1480, 1382, 1305, 1211, 1087; MS $\mathrm{m} / \mathrm{z}$ (relative intensity) $285(\mathrm{M}+2)^{+}(18), 284(\mathrm{M}+1)^{+}(100)$. HRMS calcd for $\mathrm{C}_{16} \mathrm{H}_{34} \mathrm{~N}_{3} \mathrm{O}$ 284.2696, found 284.2694.

1-(tert-Butylimino-methyl)-1,3-dimethyl-3-prop-2-ynyl-urea (9c). Colorless oil: ${ }^{1} \mathrm{H}$ NMR $\left(\mathrm{CDCl}_{3}\right) \square 1.20$ (s, 9H), 2.30 (br s, 1H), 2.96 (s, 3H), 3.11 (s, 3H), $3.98(\mathrm{~s}, 2 \mathrm{H}), 8.01$ (br s, 1H); ${ }^{13} \mathrm{C} \mathrm{NMR}\left(\mathrm{CDCl}_{3}\right) \square 31.1,32.6,36.5,40.5,55.1,73.1,79.1,147.1,161.7$; IR (thin film, $\mathrm{cm}^{-1}$ ) 3266, 2967, 1659, 1478, 1374, 1303, 1209, 1109; MS m/z (relative intensity) $211(\mathrm{M}+2)^{+}(14)$, $210(\mathrm{M}+1)^{+}(100)$. HRMS calcd for $\mathrm{C}_{10} \mathrm{H}_{20} \mathrm{~N}_{3} \mathrm{O} 210.1601$, found 210.1604. Anal. Calcd for $\mathrm{C}_{10} \mathrm{H}_{19} \mathrm{~N}_{3} \mathrm{O}$ : C, 63.13; H, 9.15, N, 20.08. Found: C, 63.10; H, 9.19, N, 20.12.

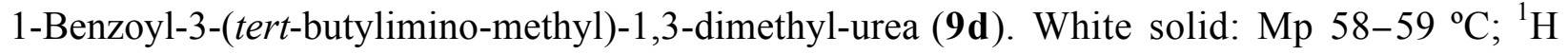

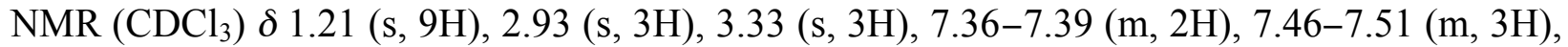
7.98 (br s, $1 \mathrm{H}) ;{ }^{13} \mathrm{C} \mathrm{NMR}\left(\mathrm{CDCl}_{3}\right) \square 30.5,34.4,55.7,128.1,128.9,132.3,135.2,143.8,162.2$, 171.5; IR (thin film, $\mathrm{cm}^{-1}$ ) 2969, 1654, 1436, 1316, 1212, 1084; MS $m / z$ (relative intensity) $187(\mathrm{M}+2)^{+}(10), 186(\mathrm{M}+1)^{+}(100)$. HRMS calcd for $\mathrm{C}_{15} \mathrm{H}_{22} \mathrm{~N}_{3} \mathrm{O}_{2}$ 276.1706, found 276.1712.

6) Hammouda, H. A.; Abd-Allah, S. O.; Sharaf, M. A. F. Egypt. J. Chem. 1989, 30, $239 \square 247$. 
1-(Benzylimino-methyl)-1,3,3-trimethyl-urea (9e). Colorless oil: ${ }^{1} \mathrm{H}$ NMR $\left(\mathrm{CDCl}_{3}\right) \square 2.90$ (s, $3 \mathrm{H}), 2.99(\mathrm{~s}, 3 \mathrm{H}), 3.07(\mathrm{~s}, 3 \mathrm{H}), 4.57(\mathrm{~s}, 2 \mathrm{H}), 7.26 \square 7.33(\mathrm{~m}, 5 \mathrm{H}), 8.63(\mathrm{br} \mathrm{s}, 1 \mathrm{H}) ;{ }^{13} \mathrm{C} \mathrm{NMR}$ $\left(\mathrm{CDCl}_{3}\right) \square 30.0,38.6,38.8,60.2,127.7,128.7,140.8,152.3,163.4$; IR (thin film, $\left.\mathrm{cm}^{-1}\right) 3431$, 2935, 1660, 1546, 1489, 1382, 1318, 1120, 1073; MS m/z (relative intensity) $221(\mathrm{M}+2)^{+}(15)$, $220(\mathrm{M}+1)^{+}(100)$. HRMS calcd for $\mathrm{C}_{12} \mathrm{H}_{18} \mathrm{~N}_{3} \mathrm{O} 220.1444$, found 220.1445.

1- $\left\{(\mathrm{E})-\left[(\right.\right.$ Benzyloxy)imino]methyl $\}-1,3,3$-trimethyl-urea (9f). Colorless oil: ${ }^{1} \mathrm{H}$ NMR $\left(\mathrm{CDCl}_{3}\right) \square$ $2.86(\mathrm{~s}, 6 \mathrm{H}), 3.04(\mathrm{~s}, 3 \mathrm{H}), 4.96(\mathrm{~s}, 2 \mathrm{H}), 7.29 \square 7.36(\mathrm{~m}, 5 \mathrm{H}), 8.26(\mathrm{br} \mathrm{s}, 1 \mathrm{H}) ;{ }^{13} \mathrm{C} \mathrm{NMR}\left(\mathrm{CDCl}_{3}\right) \square$ 33.4, 39.0, 76.4, 128.3, 128.8, 128.9, 138.0, 148.9, 160.8; IR (thin film, $\mathrm{cm}^{-1}$ ) 3503, 2936, 1672 , $1625,1494,1444,1370,1262,1128,1055 ; \mathrm{MS} m / z$ (relative intensity) $237(\mathrm{M}+2)^{+}(14), 236$ $(\mathrm{M}+1)^{+}(100)$. HRMS calcd for $\mathrm{C}_{12} \mathrm{H}_{18} \mathrm{~N}_{3} \mathrm{O}_{2} 236.1393$, found 236.1392.

1-Hexanoyl-3-\{(E)-[(benzyloxy)imino]methyl $\}$-1,3-dimethyl-urea (9g). Colorless oil: ${ }^{1} \mathrm{H}$ NMR $\left(\mathrm{CDCl}_{3}\right) \square 0.89(\mathrm{t}, J=7.0 \mathrm{~Hz}, 3 \mathrm{H}), 2.28(\mathrm{~m}, 6 \mathrm{H}), 2.35(\mathrm{~m}, 2 \mathrm{H}), 3.20(\mathrm{~s}, 3 \mathrm{H}), 3.09(\mathrm{~s}, 3 \mathrm{H}), 5.01$ $(\mathrm{s}, 2 \mathrm{H}), 7.26 \square 7.39(\mathrm{~m}, 5 \mathrm{H}), 8.36(\mathrm{br} \mathrm{s}, 1 \mathrm{H}) ;{ }^{13} \mathrm{C} \mathrm{NMR}\left(\mathrm{CDCl}_{3}\right) \square 14.3,22.8,25.0,31.7,32.2$, $33.8,35.4,77.1,128.5,128.9,129.0,137.4,145.6,157.3,174.4$; IR (thin film, $\mathrm{cm}^{-1}$ ) 2955, 2932, 2861, 1697, 1620, 1459, 1352, 1292, 1097, 1037; MS m/z (relative intensity) $342(\mathrm{M}+\mathrm{Na})^{+}$ (100), $321(\mathrm{M}+2)^{+}(4), 320(\mathrm{M}+1)^{+}(15)$. HRMS calcd for $\mathrm{C}_{17} \mathrm{H}_{26} \mathrm{~N}_{3} \mathrm{O}_{3} 320.1969$, found 320.1970 .

1-[(E)-(Methoxyimino)methyl]-1,3,3-trimethyl-urea (9h). Colorless oil: ${ }^{1} \mathrm{H}$ NMR $\left(\mathrm{CDCl}_{3}\right) \square 2.88$ (s, 6H), 3.05 (s, 3H), 3.74 (s, 3H), 8.20 (br s, 1H); ${ }^{13} \mathrm{C} \mathrm{NMR}\left(\mathrm{CDCl}_{3}\right) \square 32.3,37.6,60.7,147.2$, 159.3; IR (thin film, $\mathrm{cm}^{-1}$ ) 3514, 2944, 1663, 1626, 1502, 1384, 1270, 1129, 1056; MS m/z (relative intensity) $261(\mathrm{M}+2)^{+}(8), 260(\mathrm{M}+1)^{+}(100)$. HRMS calcd for $\mathrm{C}_{6} \mathrm{H}_{14} \mathrm{~N}_{3} \mathrm{O}_{2}$ 160.1080, found 160.1083. Anal. Calcd for $\mathrm{C}_{6} \mathrm{H}_{13} \mathrm{~N}_{3} \mathrm{O}_{2}$ : C, 45.27; $\mathrm{H}, 8.23, \mathrm{~N}, 26.40$. Found: $\mathrm{C}, 45.29 ; \mathrm{H}$, 8.30, N, 26.48.

1-Benzoyl-3-[(E)-(methoxyimino)methyl]-1,3-dimethyl-urea (9i). Colorless oil: ${ }^{1} \mathrm{H}$ NMR $\left(\mathrm{CDCl}_{3}\right) \square 2.93(\mathrm{~s}, 3 \mathrm{H}), 3.29(\mathrm{~s}, 3 \mathrm{H}), 3.69(\mathrm{~s}, 3 \mathrm{H}), 7.36 \square 7.39(\mathrm{~m}, 2 \mathrm{H}), 7.46 \square 7.51(\mathrm{~m}, 3 \mathrm{H}), 8.22$ (br s, $1 \mathrm{H}) ;{ }^{13} \mathrm{C}$ NMR $\left(\mathrm{CDCl}_{3}\right) \square 32.2,34.6,62.5,127.9,129.0,132.4,135.1,144.8,157.4,171.4$; IR (thin film, $\mathrm{cm}^{-1}$ ) 2949, 1688, 1433, 1359, 1178, 1068; MS m/z (relative intensity) $273(\mathrm{M}+2)^{+}$ (16), $272(\mathrm{M}+1)^{+}$(100). HRMS calcd for $\mathrm{C}_{12} \mathrm{H}_{16} \mathrm{~N}_{3} \mathrm{O}_{3}$ 250.1186, found 250.1189. Anal. Calcd for $\mathrm{C}_{12} \mathrm{H}_{15} \mathrm{~N}_{3} \mathrm{O}_{3}$ : C, 57.82; H, 6.07, N, 16.86. Found: C, 57.83; H, 6.09, N, 16.85.

1-(tert-Butylimino)-3-hexyl-1-methyl-urea (10a). White solid: Mp $63 \square 64{ }^{\circ} \mathrm{C} ;{ }^{1} \mathrm{H}$ NMR $\left(\mathrm{CDCl}_{3}\right)$ $\square 0.94(\mathrm{t}, J=6.3 \mathrm{~Hz}, 3 \mathrm{H}), 1.26(\mathrm{~s}, 9 \mathrm{H}), 1.27 \square 1.39(\mathrm{~m}, 7 \mathrm{H}), 1.57 \square 1.60(\mathrm{~m}, 1 \mathrm{H}), 3.20(\mathrm{~s}, 2 \mathrm{H}), 3.36$ $(\mathrm{t}, J=6.3 \mathrm{~Hz}, 2 \mathrm{H}), 7.2(\mathrm{~s}, 0.5 \mathrm{H}), 7.70(\mathrm{~s}, 0.5 \mathrm{H}) ;{ }^{13} \mathrm{C} \mathrm{NMR}\left(\mathrm{CDCl}_{3}\right) \square 14.4,23.0,27.2,29.9,30.8$, 31.9, 40.3, 55.1, 178.8, 156.6; IR (thin film, $\mathrm{cm}^{-1}$ ) 3347, 2958, 2928, 2864, 1666, 1546, 1462, 1321, 1210, 1076; MS m/z (relative intensity) $243(\mathrm{M}+2)^{+}(17), 242(\mathrm{M}+1)^{+}(100)$. HRMS calcd for $\mathrm{C}_{13} \mathrm{H}_{27} \mathrm{~N}_{3} \mathrm{O}$ 242.2227, found 242.2229.

1-(tert-Butylimino-methyl)-3-phenyl-1-methyl-urea (10b) Colorless oil: ${ }^{1} \mathrm{H}$ NMR $\left(\mathrm{CDCl}_{3}\right) \square$ $1.29(\mathrm{~s}, 9 \mathrm{H}), 3.23(\mathrm{~s}, 3 \mathrm{H}), 7.04 \square 7.06(\mathrm{~m}, 1 \mathrm{H}), 7.28 \square 7.32(\mathrm{~m}, 2 \mathrm{H}), 7.52 \square 7.54(\mathrm{~m}, 2 \mathrm{H}), 7.66(\mathrm{~s}$, $1 \mathrm{H}) ;{ }^{13} \mathrm{C} \mathrm{NMR}\left(\mathrm{CDCl}_{3}\right) \square 30.8,35.3,55.5,120.1,123.5,129.3,139.5,149.1,153.9$; IR (thin film, $\mathrm{cm}^{-1}$ ) 3316, 2964, 1669, 1596, 1549, 1448, 1317, 1237, 1076; MS $m / z$ (relative intensity) 
$256(\mathrm{M}+\mathrm{Na})^{+}(100), 235(\mathrm{M}+2)^{+}(6), 234(\mathrm{M}+1)^{+}(14)$. HRMS calcd for $\mathrm{C}_{13} \mathrm{H}_{20} \mathrm{~N}_{3} \mathrm{O} 234.1601$, found 234.1603 .

1-[(E)-(Methoxyimino)methyl]-3-phenyl-1-methyl-urea (10c). Gummy syrup: ${ }^{1} \mathrm{H}$ NMR $\left(\mathrm{CDCl}_{3}\right)$ $\square 3.21(\mathrm{~s}, 3 \mathrm{H}), 3.85(\mathrm{~s}, 3 \mathrm{H}), 7.10(\mathrm{~m}, 1 \mathrm{H}), 7.30 \square 7.34(\mathrm{~m}, 2 \mathrm{H}), 7.47 \square 748(\mathrm{~m}, 2 \mathrm{H}), 8.14(\mathrm{~s}, 1 \mathrm{H})$, 9.61 (br s, $1 \mathrm{H}) ;{ }^{13} \mathrm{C}$ NMR $\left(\mathrm{CDCl}_{3}\right) \square 34.9,62.6,120.7,124.4,129.4,138.5,148.2,152.7$; IR (thin film, $\left.\mathrm{cm}^{-1}\right) 3319,3058,2947,2236,1672,1609,1531,1451,1360,1307,1243,1162,1065$; MS $m / z$ (relative intensity) $230(\mathrm{M}+\mathrm{Na})^{+}(100), 209(\mathrm{M}+2)^{+}(4), 208(\mathrm{M}+1)^{+}(11)$. HRMS calcd for $\mathrm{C}_{10} \mathrm{H}_{14} \mathrm{~N}_{3} \mathrm{O}_{2}$ 208.1080, found 208.1081. Anal. Calcd for $\mathrm{C}_{10} \mathrm{H}_{13} \mathrm{~N}_{3} \mathrm{O}_{2}$ : C, 57.96; $\mathrm{H}, 6.32, \mathrm{~N}$, 20.28. Found: C, 57.99; H, 6.32, N, 20.31.

1-[(E)-(Methoxyimino)methyl]-3-(4-bromo-phenyl)-1-methyl-urea (10d). White solid: Mp

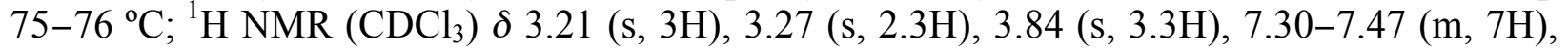
8.06 (s, 1H), 9.89 (br s, $1 \mathrm{H}) ;{ }^{13} \mathrm{C} \mathrm{NMR}\left(\mathrm{CDCl}_{3}\right) \square 34.3,35.0,62.6,120.4,120.6,120.7,123.6$, 124.4, 125.6, 129.4, 136.8, 138.5, 148.2, 150.2, 152.7; IR (thin film, $\mathrm{cm}^{-1}$ ) 3319, 2937, 2246, $1706,1612,1535,1491,1307,1253,1065 ; \mathrm{MS} m / z$ (relative intensity) $309(\mathrm{M}+\mathrm{Na})^{+}(100)$, $287(\mathrm{M}+2)^{+}(5), 286(\mathrm{M}+1)^{+}(13)$. HRMS calcd for $\mathrm{C}_{10} \mathrm{H}_{13} \mathrm{BrN}_{3} \mathrm{O}_{2} 286.0186$, found 286.0191 .

2-Bromo- $N$-methyl- $N$ '-[(E)-(benzyloxyimino)methyl]-acetamide (11). A solution of the 1- $\{(E)$ [(benzyloxy)imino]methyl $\}$-1,3-dimethylurea $(221 \mathrm{mg}, 1.0 \mathrm{mmol})$ and 2,6-lutidine (71_L, 0.98 mmol) in dry $\mathrm{CH}_{2} \mathrm{Cl}_{2}(5 \mathrm{~mL}, 0.2 \mathrm{M})$ was cooled at $-78{ }^{\circ} \mathrm{C}$ under a nitrogen atmosphere and stirred for $15 \mathrm{~min}$ before the addition of bromoacetyl bromide (freshly distilled) (81_L, 0.94 $\mathrm{mmol}$ ). The reaction mixture was slowly warmed to room temperature over ca. $2 \mathrm{~h}$ and stirred for a further $24 \mathrm{~h}$. The mixture was concentrated under vacuum and partitioned with $\mathrm{CH}_{2} \mathrm{Cl}_{2}$ and aqueous saturated solution of $\mathrm{NH}_{4} \mathrm{Cl}$, washed with brine, dried $\left(\mathrm{MgSO}_{4}\right)$, filtered, concentrated and purified by column chromatography to afford the corresponding formamidine derivatives in $68 \%$ yield $(193 \mathrm{mg}[\mathbf{1 1}+\mathbf{1 2}]$, ratio $10: 1)$. Colorless oil: ${ }^{1} \mathrm{H} \mathrm{NMR}\left(\mathrm{CDCl}_{3}\right) \square 3.20(\mathrm{~s}, 3 \mathrm{H}), 3.30$ $(\mathrm{s}, 3 \mathrm{H}), 3.93(\mathrm{~s}, 4 \mathrm{H}), 5.05(\mathrm{~s}, 4 \mathrm{H}), 7.26 \square 7.38(\mathrm{~m}, 10 \mathrm{H}), 8.47$ (br s, $1 \mathrm{H}), 8.83$ (br s, $1 \mathrm{H})$; ${ }^{13} \mathrm{C} \mathrm{NMR}$ $\left(\mathrm{CDCl}_{3}\right) \square 25.5,26.2,30.1,32.5,128.4,128.6,128.9$, 137.3, 144.5, 145.3, 165.7, 166.6; IR (thin film, $\mathrm{cm}^{-1}$ ) 3034, 2940, 1685, 1621, 1444, 1356, 1293, 1088, 1044; MS $\mathrm{m} / z$ (relative intensity) 309(307) $(\mathrm{M}+\mathrm{Na})^{+}(100), 287(285)(\mathrm{M}+1)^{+}(93)$. HRMS calcd for $\mathrm{C}_{11} \mathrm{H}_{14} \mathrm{BrN}_{2} \mathrm{O}_{2} 285.0233$, found 285.0231 .

1-(2-Bromo-acetyl)-1,3-dimethyl-3-\{(E)-[(benzyloxy)imino]methyl $\})$-urea (12). [1- $\{(E)$ [(benzyloxy)imino]methyl\}-1,3-dimethylurea + bromoacetyl bromide] Colorless oil: ${ }^{1} \mathrm{H}$ NMR $\left(\mathrm{CDCl}_{3}\right) \square 3.10(\mathrm{~s}, 3 \mathrm{H}), 3.20(\mathrm{~s}, 3 \mathrm{H}), 4.08(\mathrm{~s}, 2 \mathrm{H}), 5.03(\mathrm{~s}, 2 \mathrm{H}), 7.33 \square 7.40(\mathrm{~m}, 5 \mathrm{H}), 8.35$ (br s, $1 \mathrm{H}) ;{ }^{13} \mathrm{C}$ NMR $\left(\mathrm{CDCl}_{3}\right) \square 27.4,32.6,34.5,77.1,128.6,128.8,128.9$, 137.3, 145.3, 156.7, 168.9; IR (thin film, $\mathrm{cm}^{-1}$ ) 2919, 1694, 1624, 1436, 1345, 1288, 1094, 1037; MS m/z (relative intensity) 366(364) $(\mathrm{M}+\mathrm{Na})^{+}(28), 344(342)(\mathrm{M}+1)^{+}(100)$. HRMS calcd for $\mathrm{C}_{13} \mathrm{H}_{16} \mathrm{BrN}_{3} \mathrm{O}_{3} 342.0448$, found 342.0445 .

The identity of compound 11 was further established by reaction with thiophenol to give $N$ Methyl- $N^{\prime}-[(E)$-(benzyloxyimino)methyl)]-2-phenylsulfanyl-acetamide (14), as follows. To a solution of 2-bromo- $N$-methyl- $N^{\prime}-[(E)$-(benzyloxyimino)methyl]-acetamide (11) (284 mg, 1.0 $\mathrm{mmol})$ and $\mathrm{Et}_{3} \mathrm{~N}(142 \square \mathrm{L} 1.0 \mathrm{mmol})$ in dry $\mathrm{CH}_{2} \mathrm{Cl}_{2}(5 \mathrm{~mL}, 0.2 \mathrm{M})$ was added thiophenol (106 $\square \mathrm{L}$, $1.0 \mathrm{mmol}$ ) at $0{ }^{\circ} \mathrm{C}$ under a nitrogen atmosphere. This mixture was allowed to stir for $20 \mathrm{~min}$. 
The solution was then washed with water, dried $\left(\mathrm{MgSO}_{4}\right)$, filtered, concentrated and purified by column chromatography to give the formamidine derivative $\mathbf{1 4}$ in $92 \%$ yield $(288.8 \mathrm{mg})$.

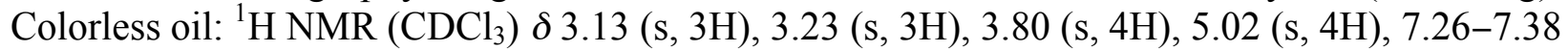
(m, 15H), 8.49 (br s, 1H), 8.84 (br s, $1 \mathrm{H}) ;{ }^{13} \mathrm{C} \mathrm{NMR}\left(\mathrm{CDCl}_{3}\right) \square 29.8,32.3,37.7,37.9,128.1$, 128.2 , 128.3, 128.6, 128.8, 128.9, 129.1, 129.6, 131.5, 131.6, 144.8, 145.6, 167.6, 168.8; IR (thin film, $\mathrm{cm}^{-1}$ ) 3059, 2924, 1683, 1619, 1445, 1364, 1300, 1099; MS $\mathrm{m} / z$ (relative intensity) 337 $(\mathrm{M}+\mathrm{Na})^{+}(63), 316(\mathrm{M}+2)^{+}(20), 315(\mathrm{M}+1)^{+}(100)$. HRMS calcd for $\mathrm{C}_{17} \mathrm{H}_{19} \mathrm{~N}_{2} \mathrm{O}_{2} \mathrm{~S} 315.1162$, found 315.1163.

Compounds 11 and 14 display two rotameric forms in their ${ }^{1} \mathrm{H}$ and ${ }^{13} \mathrm{C}$ NMR spectra, identified by coalescence upon heating, as shown below for

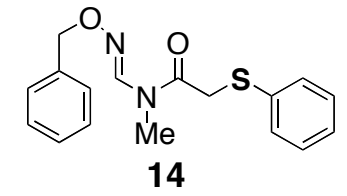
14.

NMR Spectra begin on the next page. 

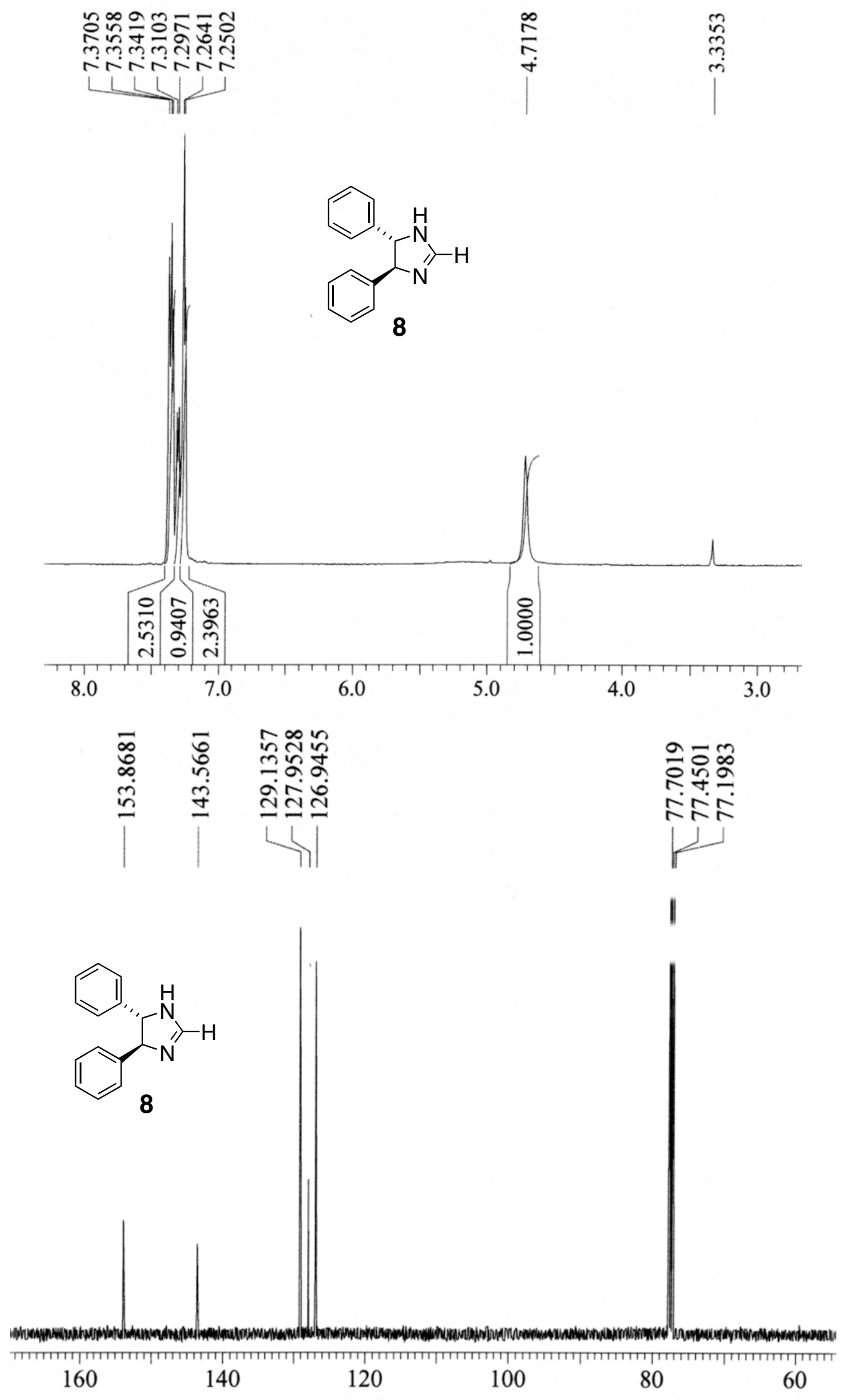

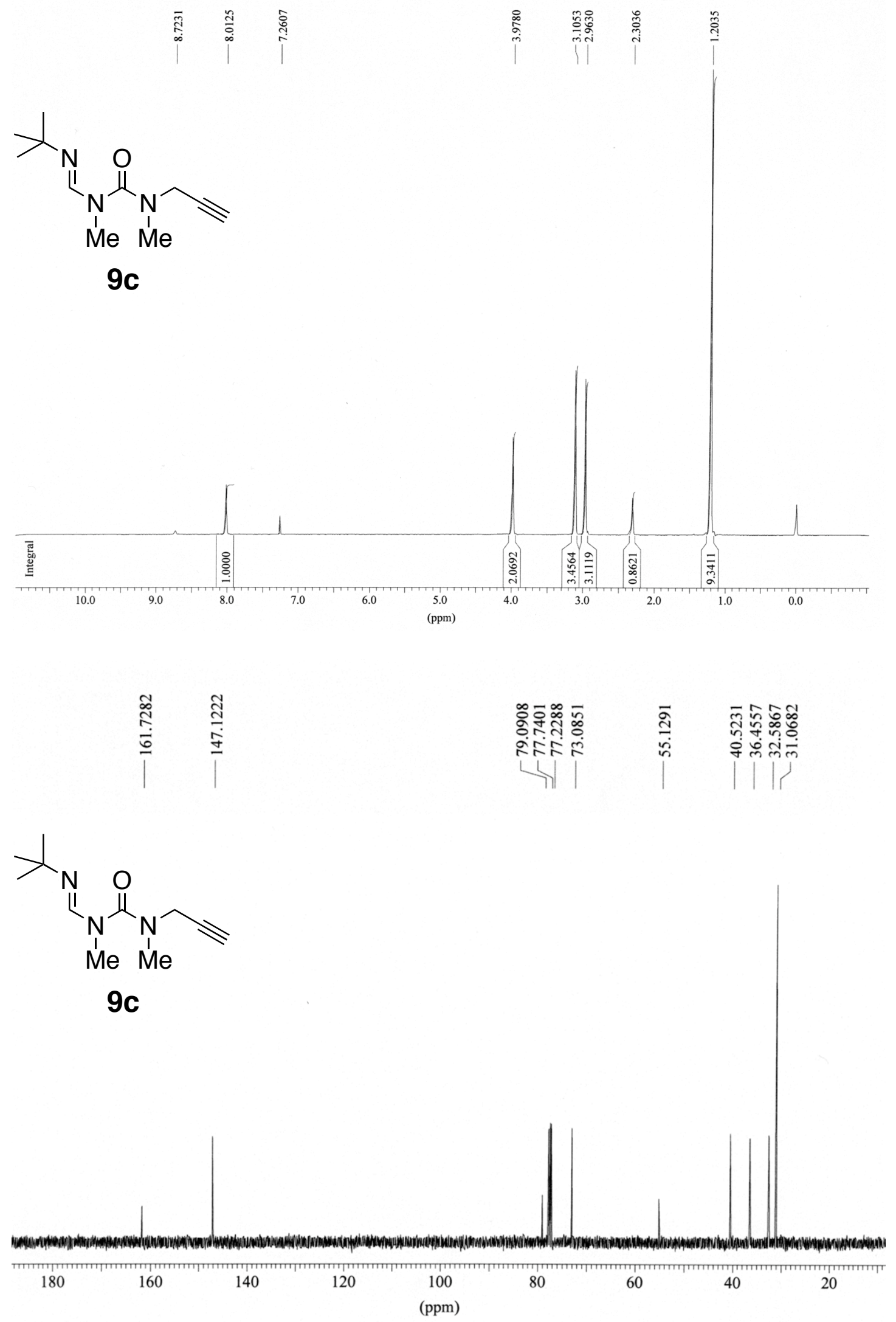

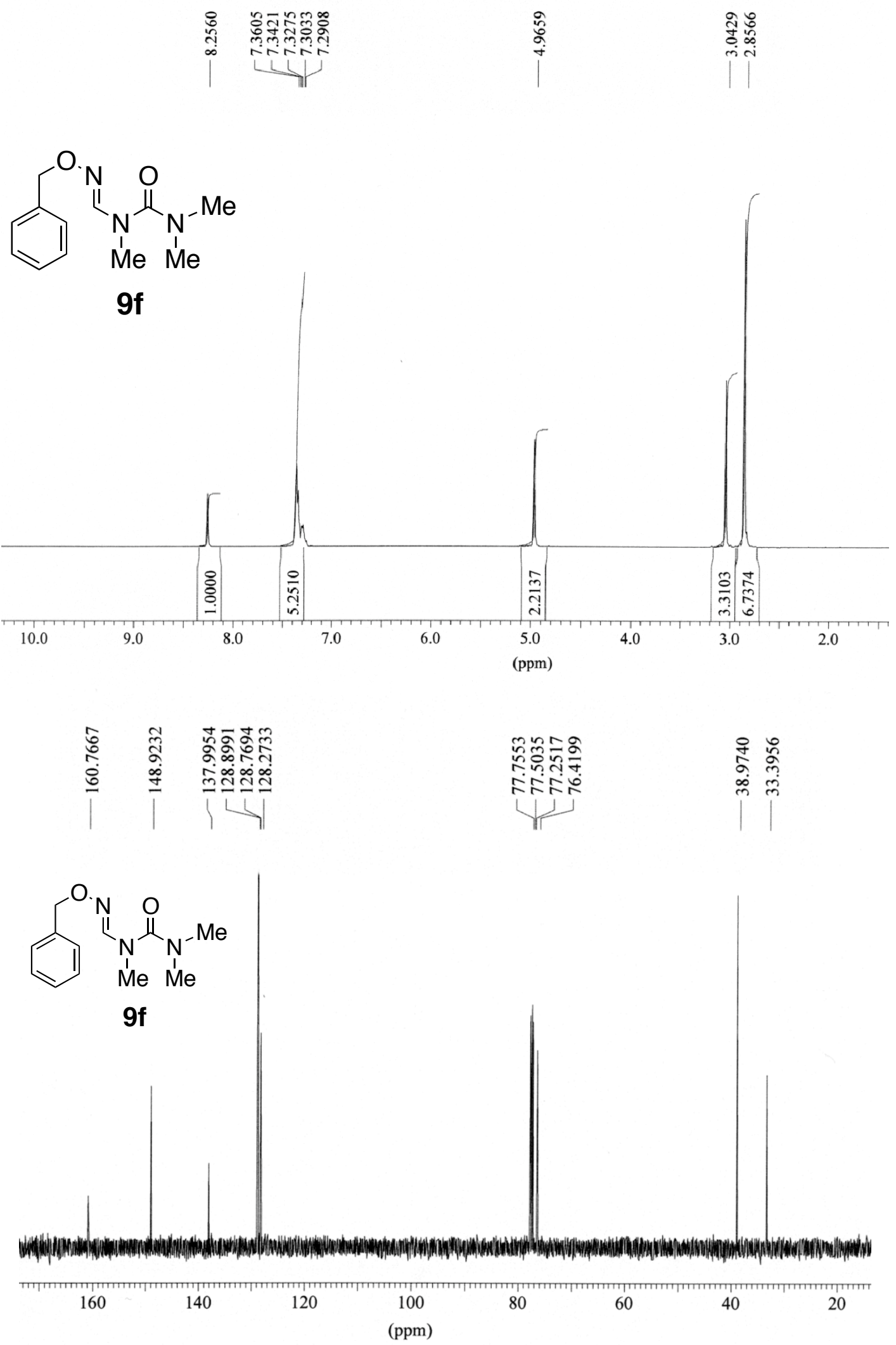

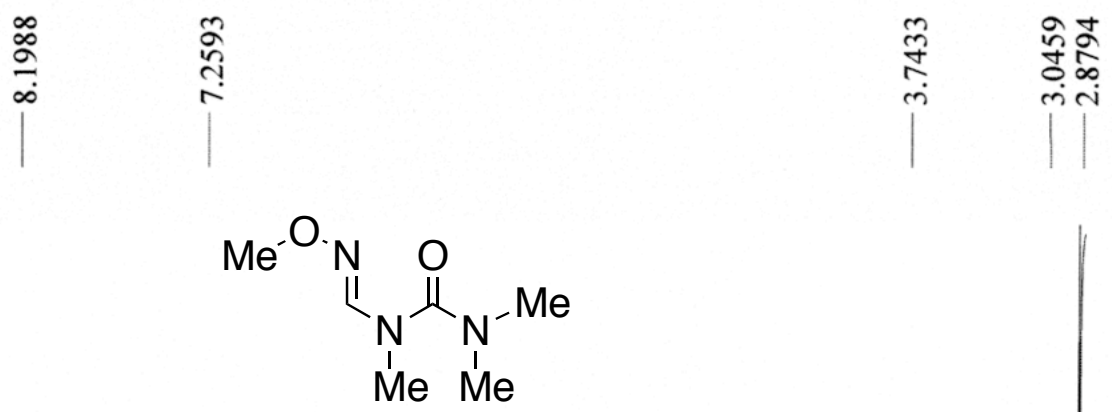

9h

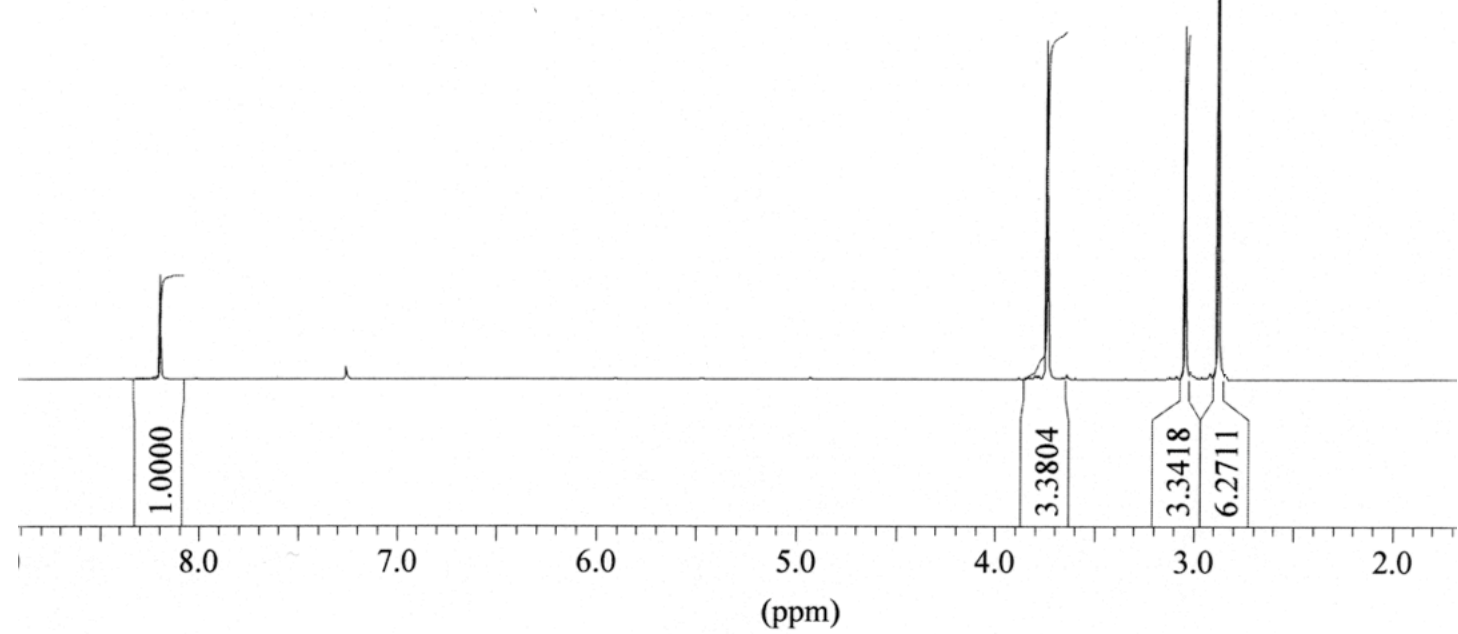

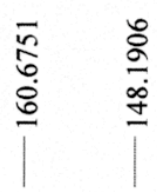
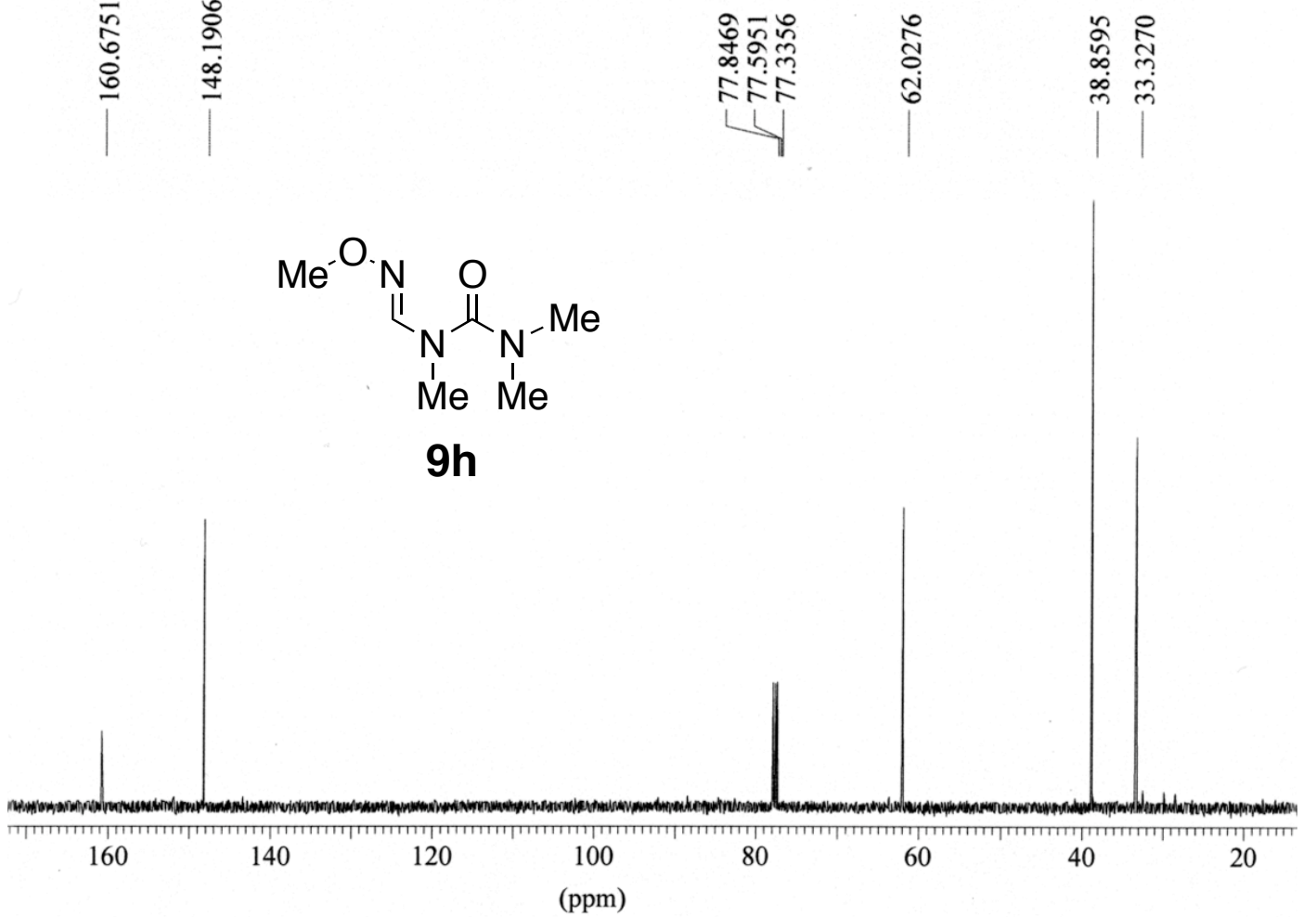

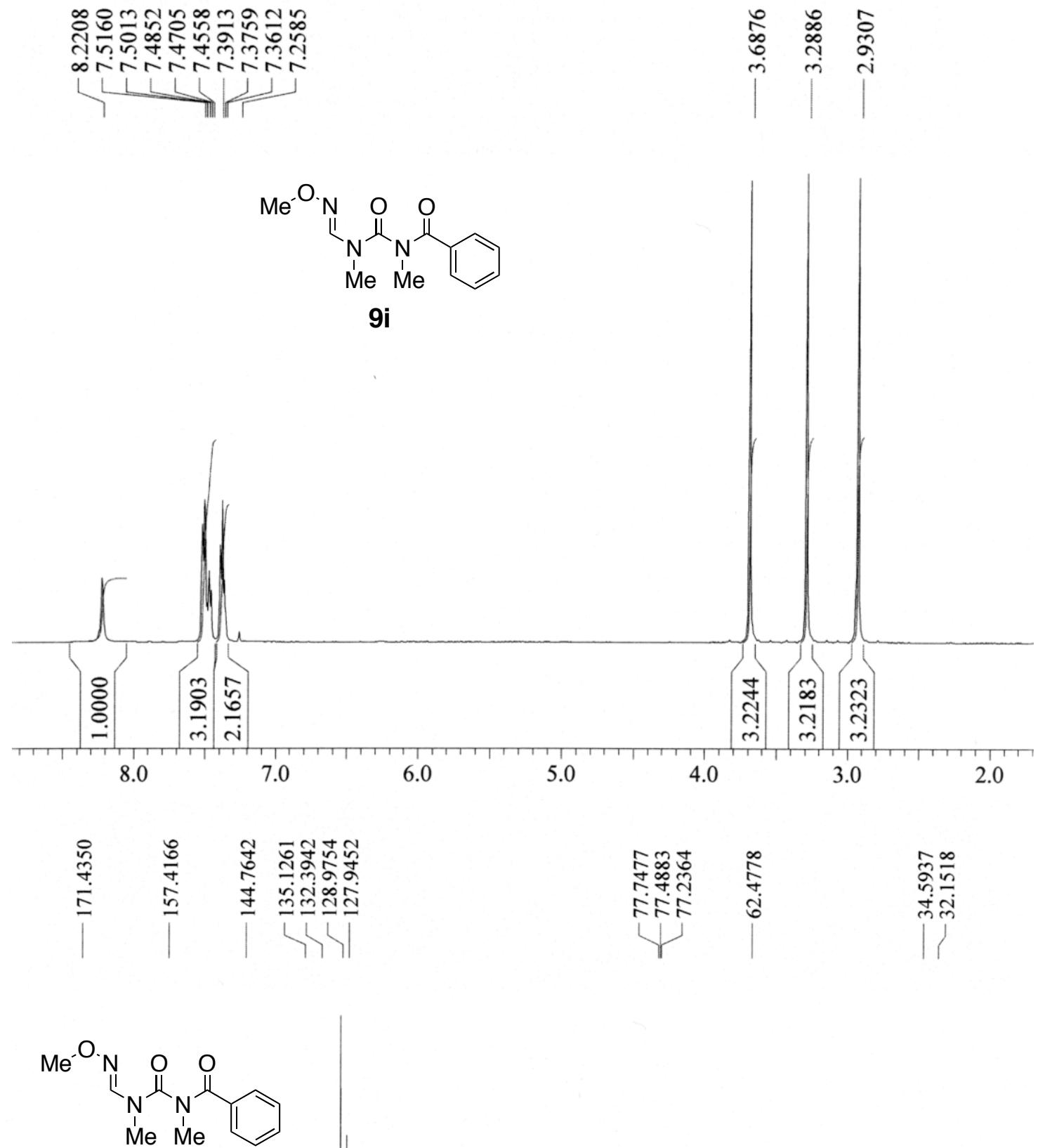

9i

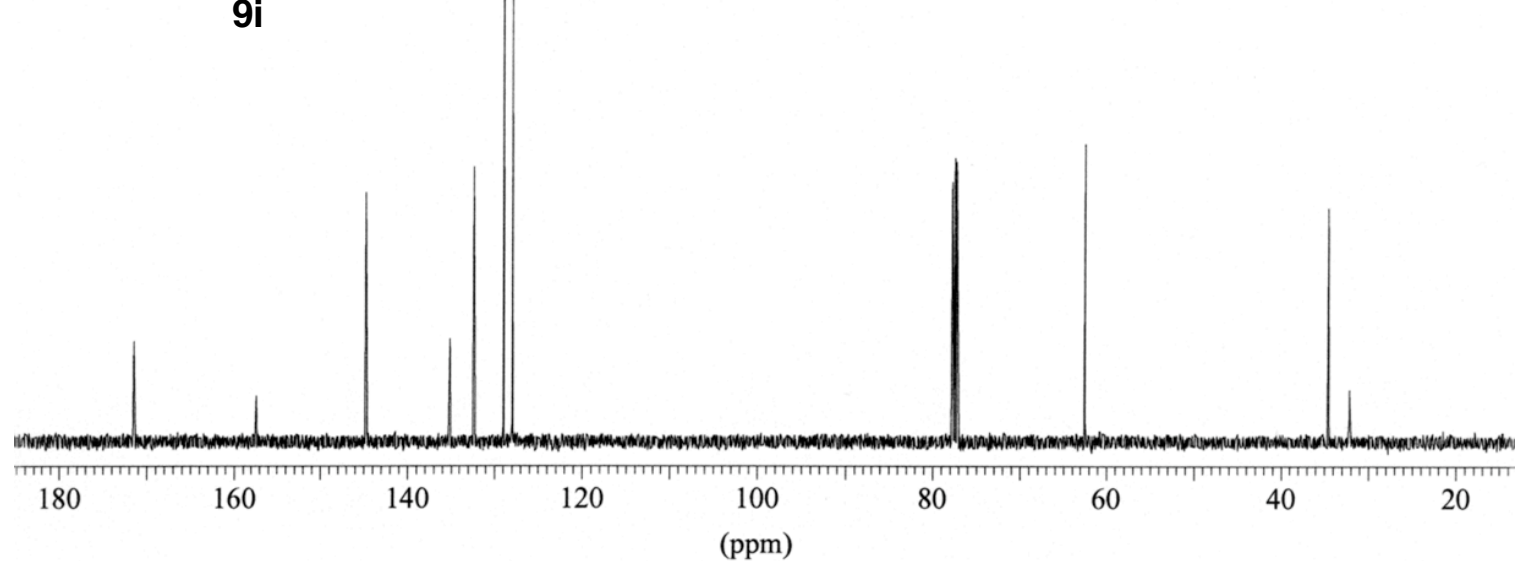




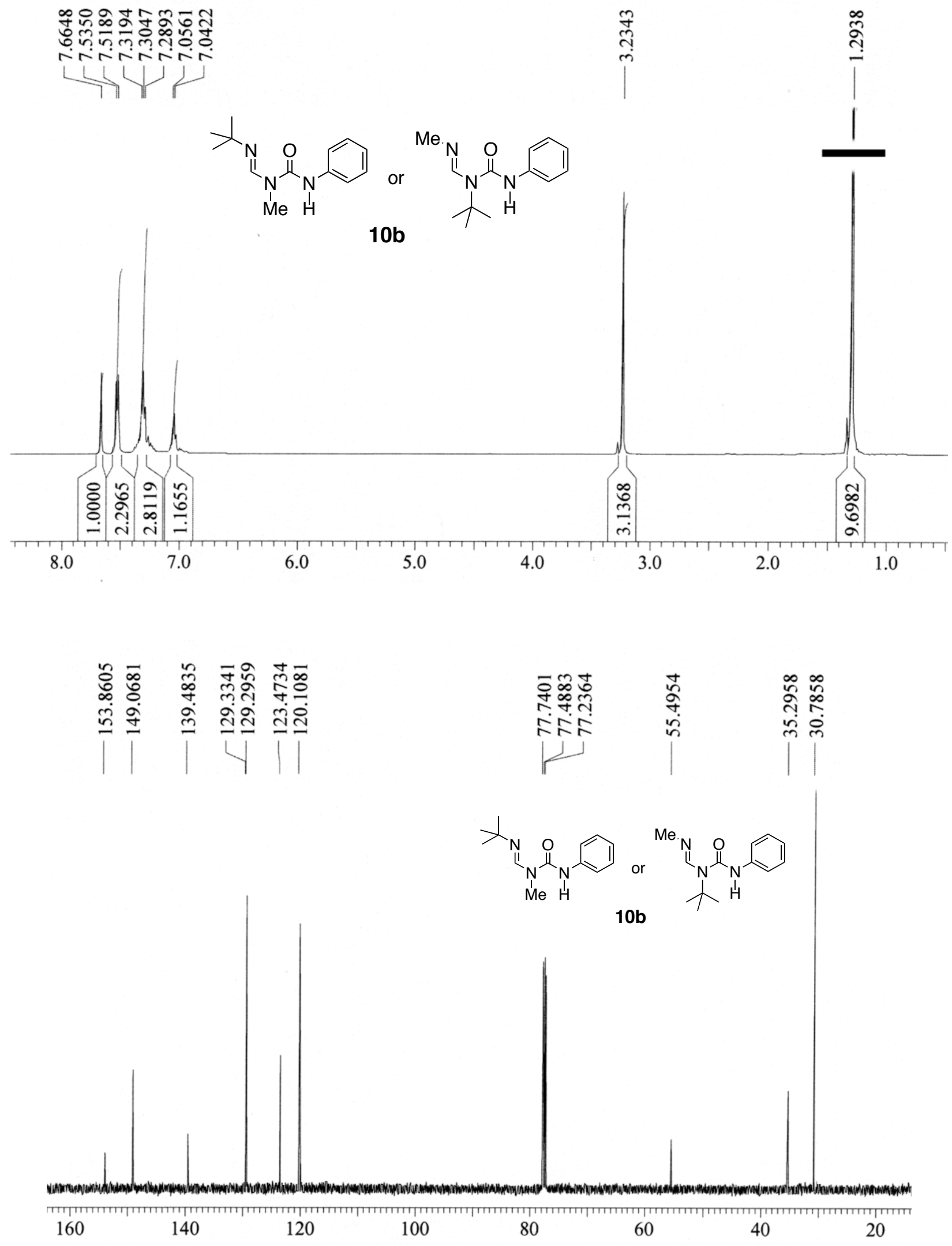



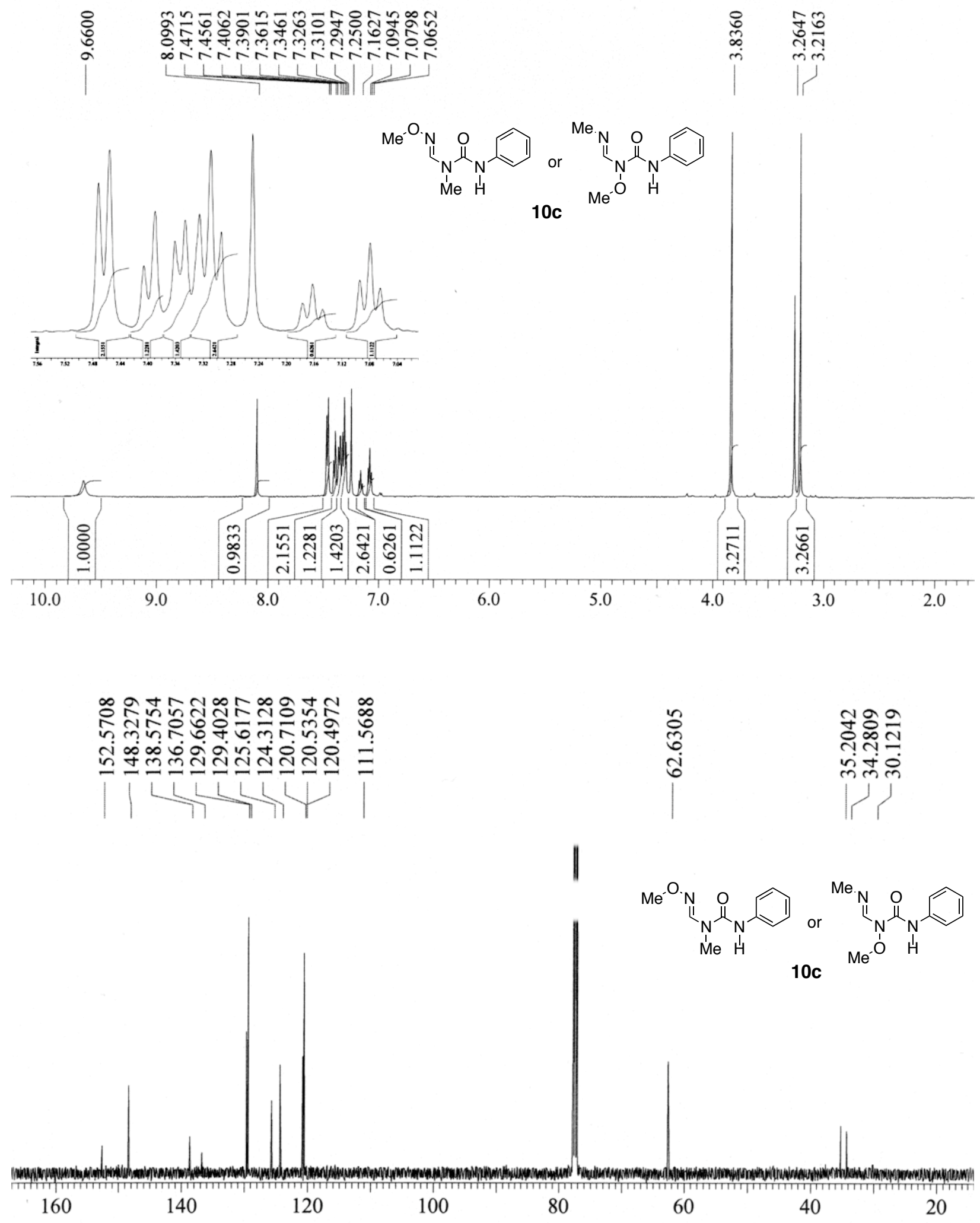

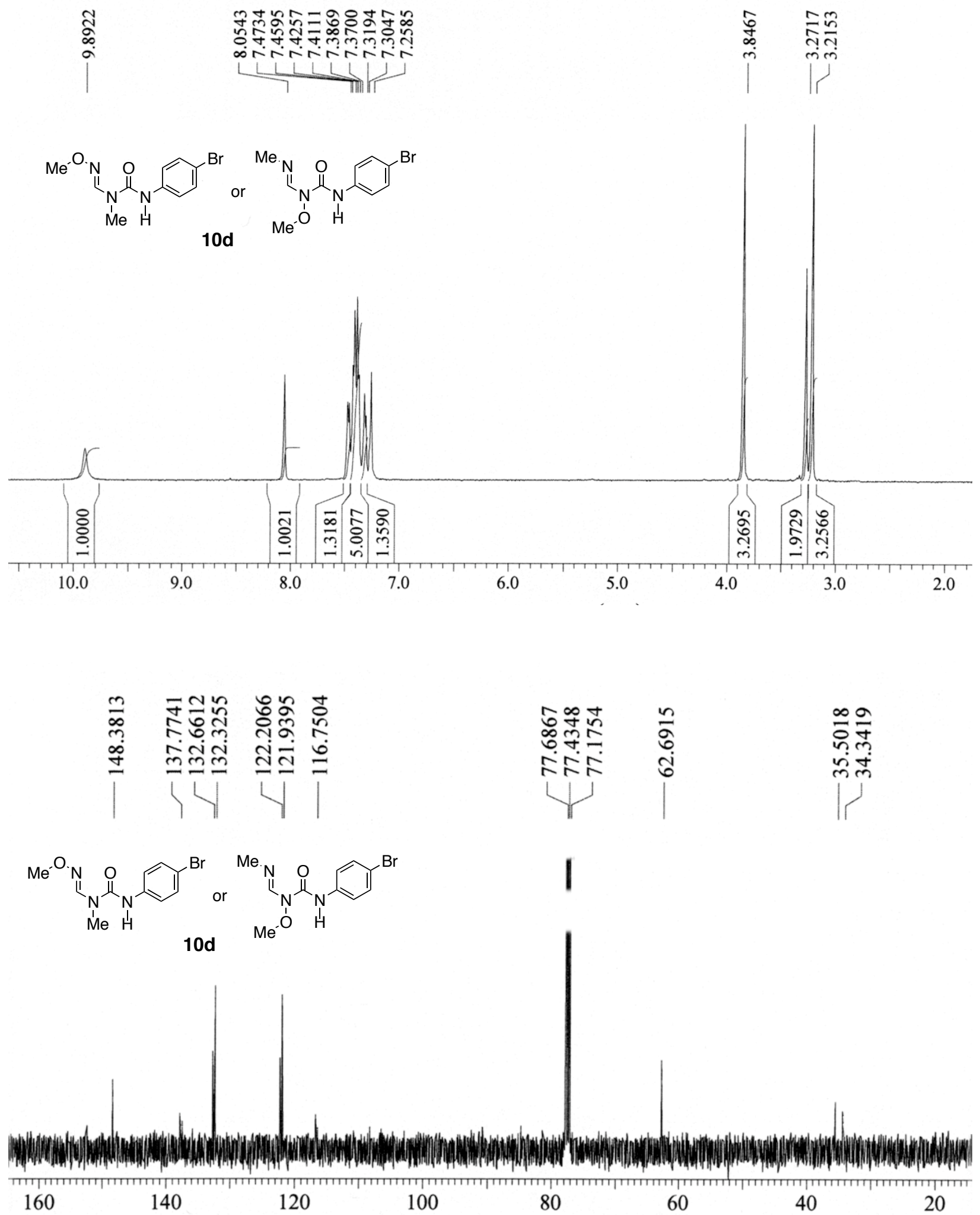


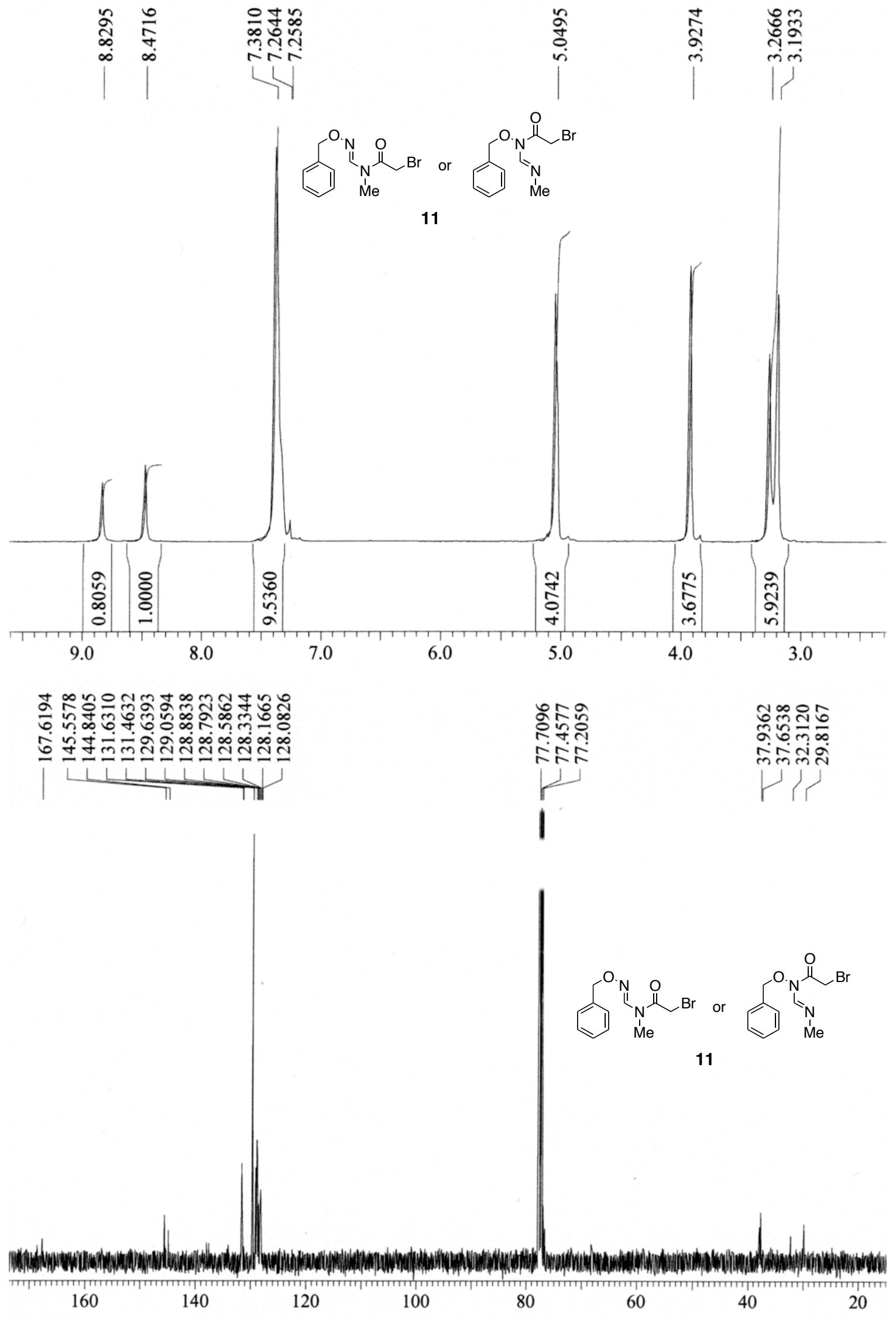




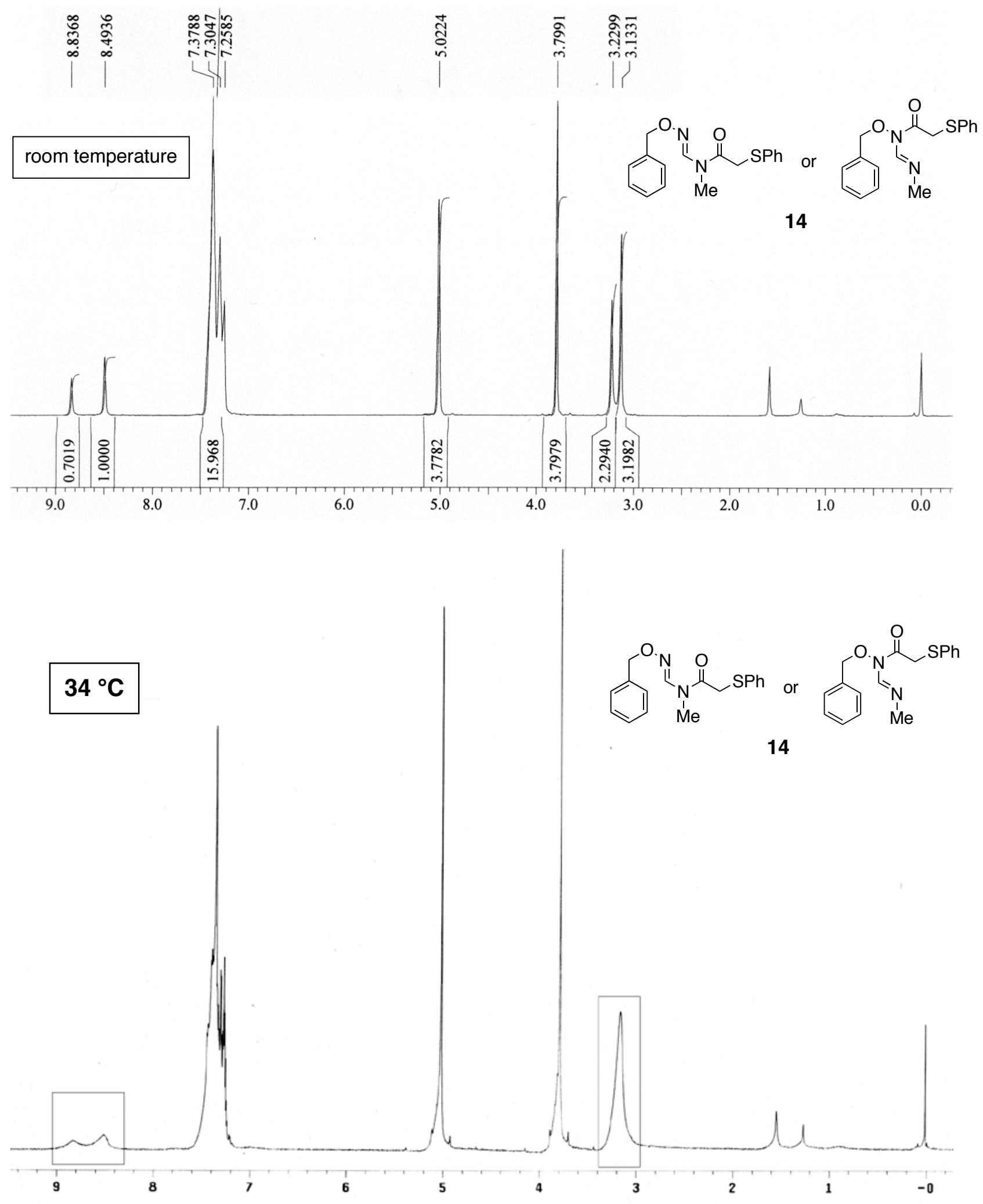




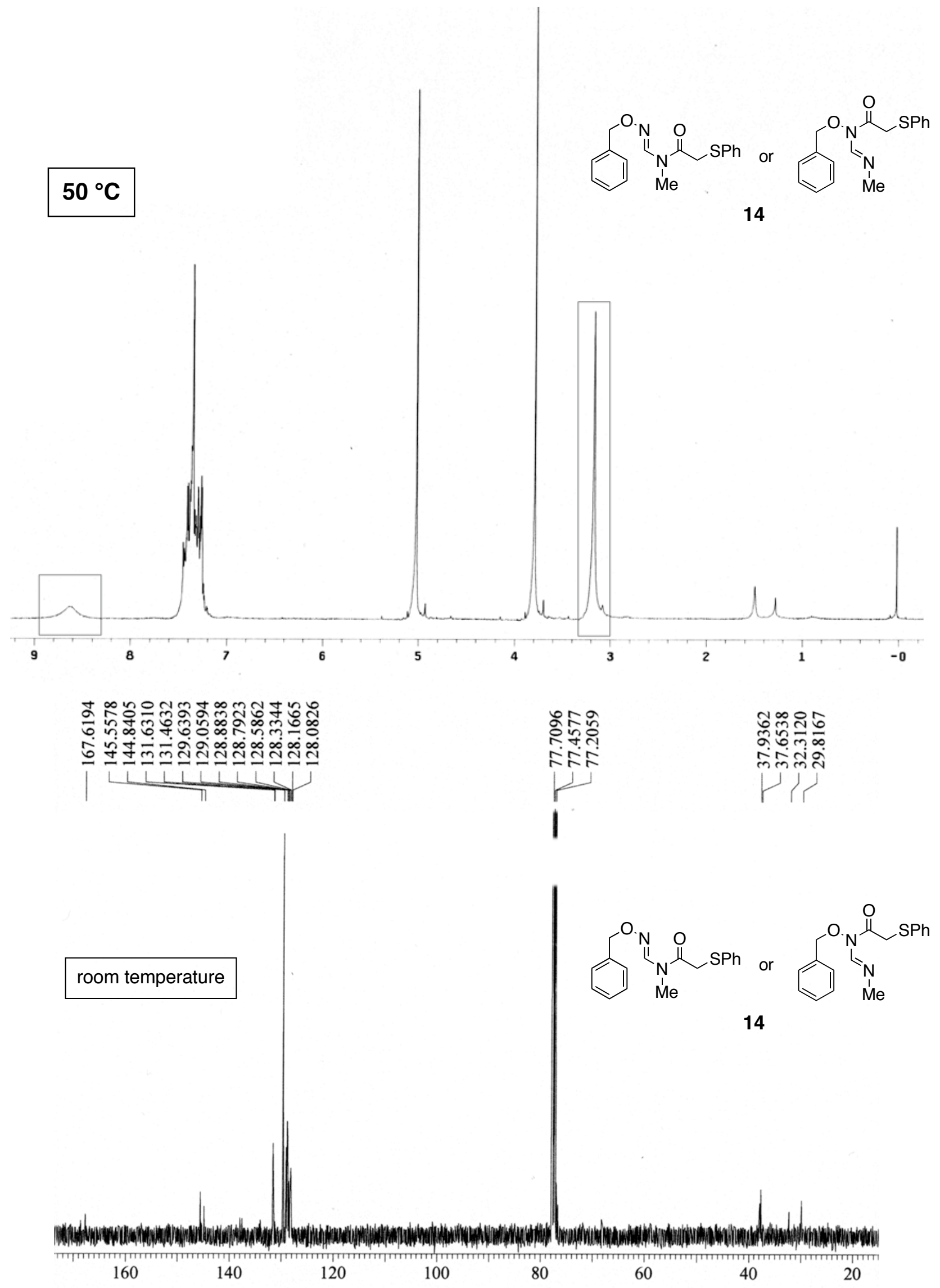

